\title{
Short and long-term effects of high-dose intravenous methylprednisolone pulse therapy on thyroid-associated ophthalmopathy
}

\author{
XIAOMEI LIU ${ }^{1,2}$, SHU WANG $^{1}$, LI QIN $^{3}$, WEI QIANG ${ }^{1}$, MAHESH DAHAL $^{1}$, \\ PING FAN ${ }^{4}$, SHAN GAO ${ }^{5}$ and BINGYIN SHI ${ }^{1}$
}

\begin{abstract}
${ }^{1}$ Department of Endocrinology, First Affiliated Hospital of Xi'an Jiaotong University, Xi'an, Shaanxi 710061;
${ }^{2}$ Department of Endocrinology, Nanjing First Hospital, Nanjing Medical University, Nanjing, Jiangsu 210006; Departments of ${ }^{3}$ Ophthalmology and ${ }^{4}$ Rheumatism, First Affiliated Hospital of Xi'an Jiaotong University; ${ }^{5}$ Department of Endocrinology, Shaanxi Provincial People's Hospital Affiliated to Xi'an Jiaotong University, Xi'an, Shaanxi 710061, P.R. China
\end{abstract}

Received March 3, 2015; Accepted April 7, 2016

DOI: $10.3892 /$ etm.2016.3446

\begin{abstract}
The majority of previous studies on high-dose intravenous methylprednisolone pulse (IVMP) therapy have observed the clinical conditions of patients prior to and following treatment without any long-term follow-up, and these studies have predominantly focused on combined treatment. The present prospective clinical study aimed to assess the long-term effects and safety of high-dose IVMP therapy in thyroid-associated ophthalmopathy (TAO), as well as the significance of thyrotropin receptor antibody (TRAb) and soluble intercellular adhesion molecule-1 (sICAM-1) during IVMP therapy. A total of 58 patients with TAO were treated with high-dose IVMP therapy, and their clinical characteristics and indices were recorded before, during and after therapy, with a 12-57 month (mean, 28.4 months) follow-up. Before treatment and on the second day after each IVMP therapy, serum TRAb and sICAM-1 levels were evaluated in 23 patients with TAO via a competitive radioimmunoassay and enzyme-linked immunosorbent assay, respectively. The results of the present study demonstrated that the symptoms of eyelid swelling, ophthalmodynia, photophobia, lacrimation and diplopia, and visual acuity, ocular motility, proptosis and clinical activity score (CAS) indices were all significantly improved after IVMP therapy. In addition, analysis of covariance demonstrated that alterations in the levels of serum TRAb during the course of treatment were associated with CAS of TAO, whereas the change in serum sICAM-1 was not. In conclusion, high-dose IVMP therapy is an effective, safe,
\end{abstract}

Correspondence to: Professor Bingyin Shi, Department of Endocrinology, First Affiliated Hospital of Xi'an Jiaotong University, 277 Yanta West Road, Xi'an, Shaanxi 710061, P.R. China

E-mail: shibingy@126.com

Key words: Graves' ophthalmopathy, methylprednisolone, pulse therapy, thyroid-associated ophthalmopathy, thyroid eye disease stable and well-tolerated treatment for TAO, which is associated with rare, minor adverse effects. Furthermore, serum TRAb levels are correlated with the CAS of TAO and may serve as a predictor of the response to methylprednisolone therapy.

\section{Introduction}

Thyroid-associated ophthalmopathy (TAO), which is also called Graves' ophthalmopathy or thyroid eye disease, is a complex inflammatory disorder of the eye that is usually associated with thyroid disorder (1). The annual incidence of TAO is $\sim 16 / 100,000$ in women and 3/100,000 in men in Olmsted county (2). Glucocorticoids are a commonly used agent in the treatment of TAO since the 1950s (3-5). Recent evidence has suggested that high-dose IVMP therapy is superior to oral glucocorticoid for the treatment of active TAO, with an excellent curative effect, quick response and fewer adverse effects. In previous studies, IVMP has predominantly been used in combination with other treatments, and only the short term effects (before and immediately after treatment) have been observed. No definite IVMP therapy was recommended in the consensus statement of the European Group on Graves' Orbitopathy (EUGOGO), as evidence for the superiority of any of the different IVMP schedules is lacking (6).

The aim of the present study was to prospectively evaluate the long-term effects of high-dose IVMP therapy in a cohort of patients with moderately to severely active TAO. Additionally, as serum TRAb has a vital role in the occurrence and progression of TAO $(7,8)$ and SICAM-1 is as an indicator of the progress of TAO and a predictor of its prognosis $(9,10)$, serum TRAb and sICAM-1 were evaluated in 23 patients with TAO.

\section{Materials and methods}

Experimental design. The present prospective clinical study was performed to evaluate the long-term effects and safety of high-dose IVMP therapy on TAO. A total of 73 patients with 
TAO were recruited from the Department of Endocrinology at the First Affiliated Hospital of Xi'an Jiaotong University (Xi'an, China) between October 2006 and November 2010. A total of 15 participants were excluded or lost to follow-up. Informed consent was obtained from the remaining 58 participants, or from the parents or legal guardians of minors or incapacitated adults. The present protocol was approved by the local ethical committee.

Inclusion criteria. Diagnosis of TAO was defined by the presence of typical clinical features, including protruding and watery eyes, retrobulbar pain, photophobia, and double and blurred vision, and detailed ophthalmological examination, including exophthalmometry, measurement of eye movements, eyelid retraction and tonometry (11). In addition, a routine orbital computed tomography scan (Philips Brilliance 64 CT scanner, Philips Medical Systems, Inc., Bothell, WA, USA) was performed for all patients before commencement of any systemic medical treatment to evaluate the extraocular muscles and exclude the orbital space-occupying lesions.

Moderate-to-severe TAO with a clinical activity score $(\mathrm{CAS}) \geq 4$ is defined as moderately to severely active TAO. The CAS is based on four classical signs (pain, redness, swelling and impaired function) which were proposed by Mourits et al (12) and consists of 10 items. These are the following: a painful, oppressive feeling on or behind the globe; pain on attempting an up, side or down gaze; redness of the eyelids; diffuse redness of the conjuctiva; chemosis; swollen caruncle; oedema of the eyelid(s); increase of proptosis by $\geq 2 \mathrm{~mm}$ during a period between 1 and 3 months; decrease in visual acuity of one or more times on the Snellen chart during 1 and 3 months and a decrease in eye movements in any direction $\geq 5^{\circ}$ during a period between 1 and 3 months. TAO is defined as active eye disease when CAS is $\geq 4$, otherwise it is classified as inactive eye disease. Moderate-to-severe TAO has a sufficient impact on the daily life of patients, including any one or more of the following symptoms: Lid retraction $\geq 2 \mathrm{~mm}$, moderate or severe soft tissue involvement, exophthalmos $\geq 3 \mathrm{~mm}$ above normal for race and gender and inconstant or constant diplopia (6).

Patients. A total of 73 patients who suffered from moderately-to-severely active TAO were recruited for the present study. A total of 15 patients were not included. Three patients with serious phthisis or hepatitis were excluded prior to enrollment and five patients were lost during follow-up. Two patients received oral glucocorticoids during the course of high-dose IVMP therapy. One patient only underwent one cycle of IVMP therapy due to a relapse of hepatitis, and four patients received other treatment after IVMP therapy, including retrobulbar injection of triamcinolone acetonide $(n=2)$, surgery $(n=2)$ and 99Tc-MDP therapy $(n=2)$. The remaining 58 patients received high-dose IVMP therapy, and six participants underwent subsequent orbital irradiation after high-dose IVMP therapy due to unsatisfying effects. The levels of serum TRAb and sICAM-1 were evaluated in 23 patients who received three cycles of pulse therapy. Follow-up was conducted for 12-57 months after the therapy, with a mean of 28.4 months. A summary of the clinical characteristics of the patients is shown in Table I.
Treatment. According to age, weight, degree of illness and the presence of comorbidities, high-dose IVMP therapy was administered. IVMP therapy composed of 0.5-1 g IV methylprednisolone three times every other day, repeated with intervals of 20 days for a total of three cycles. Additional cycles were administered in specific special and severe patients under the clinician's judgment (Table I). During the course of treatment, proton pump inhibitors or $\mathrm{H} 2$ receptor antagonists and calcium supplements were prescribed to every patient. Low-salt diet, high pillow lying and sunglasses were also recommended, whereas eye drops were only used when required in 42 patients. A total of 47 patients were administered thyroxine or antithyroid agents, depending on their thyroid function. Five patients received anti-tuberculosis drugs for at least three months due to a positive reaction to a purified protein derivative skin test; although no other evidence of infection was detected. All patients were informed of the risk of smoking for TAO and all cigarette smokers were advised to stop smoking.

Follow-up. Patients were followed-up by via face-to-face interviews, telephone calls and mail. At the end of follow-up, an appointment was made with the patients for a thorough examination of eyelid swelling, ophthalmalgia, photophobia, lacrimation, diplopia, ocular motility, visual acuity, proptosis, intraocular pressure and CAS. The first four symptoms were subjectively categorized into four grades: 0 , no; 1 , mild; 2, moderate; and 3, severe. According to the EUGOGO criteria, the severity measures of diplopia/ocular motility were as follows, 0 , no diplopia/free; 1 , intermittent (diplopia in primary position of gaze, when tired or when first awakening)/restriction of eye movement at $<4 / 6$ directions (upper right, lateral right, lower right, upper left, lateral left and lower left); 2, inconstant (diplopia at extremes of gaze)/restriction of eye movement at $\geq 4 / 6$ directions; and 3 , constant (continuous diplopia in primary or reading position)/fixation (6). Individual symptom responses were defined as a complete response if the symptom was completely resolved or as a partial response if there was an improvement in the symptom without complete resolution. Examinations of visual acuity, proptosis and intraocular pressure were conducted by an ophthalmologist and endocrinologist using the international standard vision chart, and a statometer and noncontact tonometer. Mean values were taken. Furthermore, adverse effects of glucocorticoid therapy were also recorded, including: Low bone mineral density, assessed by bone density test or symptoms; liver damage, assessed via blood test; weight gain; cushingoid features, assessed by symptoms; hypertension, assessed via blood pressure; hyperglycemia, assessed via blood glucose; peptic ulcer, assessed by symptoms; mental disorder, assessed by symptoms and serious infection, assessed by symptoms.

Levels of serum TRAb (human TRAb RIA kit, Medipan GmbH, Dahlewitz, Germany) and sICAM-1 (ELISA kit, Bender Company, Vienna, Austria) were evaluated by a competitive radioimmunoassay and enzyme-1inked immunosorbent assay, respectively, prior to treatment and on the second day following each IVMP therapy in 23 patients who received three cycles of pulse therapy.

Statistical analysis. Symptoms and the results of ophthalmological examination were compared before treatment, after 
Table I. Baseline characteristics of the 58 patients enrolled in the present study.

\begin{tabular}{lr}
\hline Characteristic & $\mathrm{N}(\%)$ \\
\hline Female & $32(55.2)$ \\
Male & $26(44.8)$ \\
Cigarette smokers & $19(32.8)$ \\
Age (years) & \\
Duration of thyroid disease (months) & \\
Duration of eye disease (months) & \\
Thyroid diseases & \\
Graves disease & $50(86.2)$ \\
Hashimoto's thyroiditis & $6(10.4)$ \\
Hypothyroidism & $1(1.7)$ \\
Euthyroidism & $1(1.7)$ \\
Thyroid function & \\
TSH $<0.25$ & $25(43.1)$ \\
$0.25 \leq$ TSH $\leq 5.0$ & $21(36.2)$ \\
TSH $>5.0$ & $12(20.7)$ \\
Serum T4 (ug/dl)
\end{tabular}

$9.1 \pm 3.8$

8.6

$1.5-19.8$

Serum T3 (ng/ml)

$1.7 \pm 0.8$

1.5

$0.6-4.8$

Previous treatment prior to study

Radioactive iodine therapy

4 (6.9)

Oral glucocorticoid

$11(19.0)$

Retrobulbar injection of triamcinolone acetonide

$2(3.4)$

Comorbidities

Diabetes

$4(6.9)$

$6(10.3)$

Impaired glucose tolerance

$11(19.0)$

Hypertension

$3(5.2)$

Duration of follow-up (months)

$12 \leq$ duration $<24$

$26(44.8)$

$46.2 \pm 13.7$

46.5

11-74

$15.8 \pm 20.0$

$0-240$

9.0

$1-108$

$24 \leq$ duration $<48$

$23(39.7)$

Duration $\geq 48$

$9(15.5)$

Patients tested for serum TRAb and sICAM-1

$23(39.7)$

Female

$14(24.1)$

$9(15.5)$

Male

$21(36.2)$

Graves disease

2 (3.4)

Hashimoto's thyroiditis

Cycles of high-dose IVMP therapy

2

8 (13.8)

3

$33(56.9)$

$11(19.0)$

3 (5.2)

2 (3.4)

$1(1.7)$

7

$28.4 \pm 13.8$

26.0

$12-57$

5

6

Cumulative doses of intravenous methylprednisolone

3.0

$2(3.4)$

4.5

$10(17.2)$

6.0

$6(10.3)$

7.5

$1(1.7)$

8.0

$2(3.4)$

9.0

$25(43.1)$

10.5

$7(12.1)$ 
Table I. Continued.

\begin{tabular}{lcc}
\hline Characteristic & Number $(\%)$ & Mean \pm SD \\
\hline 13.0 & $1(1.7)$ & \\
15.0 & $1(1.7)$ & \\
19.5 & $1(1.7)$ & \\
\hline
\end{tabular}

SD, standard deviation; TSH, thyroid stimulating hormone; TRAb, thyrotropin receptor antibody; sICAM-1, soluble intercellular adhesion molecule-1; IVMP, intravenous methylprednisolone pulse.

Table II. Response rate to IVMP over time, as determined by five clinical symptoms.

\begin{tabular}{|c|c|c|c|c|c|}
\hline \multirow[b]{2}{*}{ Symptom } & \multirow{2}{*}{$\begin{array}{c}\text { Baseline } \\
\text { No }(\%)\end{array}$} & \multicolumn{2}{|c|}{1 month after treatment } & \multicolumn{2}{|c|}{ End of follow-up } \\
\hline & & CR No $(\%)$ & PR No (\%) & CR No $(\%)$ & PR No (\%) \\
\hline Eye swelling & $50(86.2)$ & $15(30.0)$ & $23(46.0)$ & $22(44.0)$ & $19(38.0)$ \\
\hline Ophthalmodynia & $36(62.1)$ & $29(80.6)$ & $6(16.7)$ & $32(88.9)$ & $1(2.8)$ \\
\hline Photophobia & $54(93.1)$ & $16(29.6)$ & $33(61.1)$ & $28(51.9)$ & $19(35.2)$ \\
\hline Lacrimation & $52(89.7)$ & $24(46.2)$ & $23(44.2)$ & $36(69.2)$ & $8(15.4)$ \\
\hline Diplopia & $43(74.1)$ & $7(16.3)$ & $12(27.9)$ & $21(48.8)$ & $16(37.2)$ \\
\hline
\end{tabular}

CR, complete response; No, number; IVMP, intravenous methylprednisolone pulse therapy; PR, partial response.

Table III. Comparisons of laboratory indicators of proptosis over time.

\begin{tabular}{lcccr}
\hline Eye & Baseline & 1 month after treatment & End of follow-up & Reference range \\
\hline Left & $19.14 \pm 2.74$ & $18.16 \pm 2.90$ & $17.44 \pm 3.11$ & $12.00-14.00$ \\
Right & $18.82 \pm 2.56$ & $18.23 \pm 2.78$ & $17.32 \pm 3.14$ & $12.00-14.00$ \\
\hline
\end{tabular}

A total of 52 patients suffered from exophthalmos, and 104 eyes were evaluated. Data are expressed as the mean \pm standard deviation. Reference ranges are specific to healthy adults in China.

treatment (one month after IVMP) and at the end of follow-up using repeated-measures analysis of variance (ANOVA). To determine if the values differed with gender, age or smoking status, one-way repeated-measures ANOVA was used. TRAb and sICAM-1 levels were analyzed prior to and following each treatment cycle using the least significant difference t-test and analysis of covariance. $\mathrm{P}<0.05$ was considered to indicate a statistically significant difference.

\section{Results}

Remission of symptoms was achieved following treatment. Symptoms and clinical outcomes of the 58 patients are shown in Table II. The grades of the five symptoms (eyelid swelling, ophthalmodynia, photophobia, lacrimation and diplopia) were all significantly reduced one month after treatment, as compared with before treatment $(\mathrm{P}<0.001)$. No significant differences in the grades of eyelid swelling, photophobia and lacrimation were detected between one month after therapy and the end of follow-up; whereas a significant increase in the grades of ophthalmodynia $(\mathrm{P}=0.046)$ and diplopia $(\mathrm{P}<0.001)$ were detected between these time points. Dividing the 58 patients into two groups according to gender, age ( $\leq 40$ years vs. $>40$ years old) and smoking status, demonstrated that these groupings did not make a significant difference to the interaction effect in all five symptoms.

Visual acuity was improved following treatment. Among the 58 patients examined, 52 patients exhibited reduced visual acuity due to TAO prior to treatment. Visual acuity testing with the international standard vision chart (Fig. 1) revealed that visual acuity was significantly improved one month after therapy in the left eye $(\mathrm{P}=0.006)$ and right eye $(\mathrm{P}<0.001)$ and at the end of follow-up in the left eye $(\mathrm{P}=0.001)$ and right eye $(\mathrm{P}<0.001)$, as compared with before treatment. Significant improvements in visual acuity were also detected between one month after therapy and the end of follow-up in both the left $(\mathrm{P}=0.020)$ and right $(\mathrm{P}=0.004)$ eyes. 


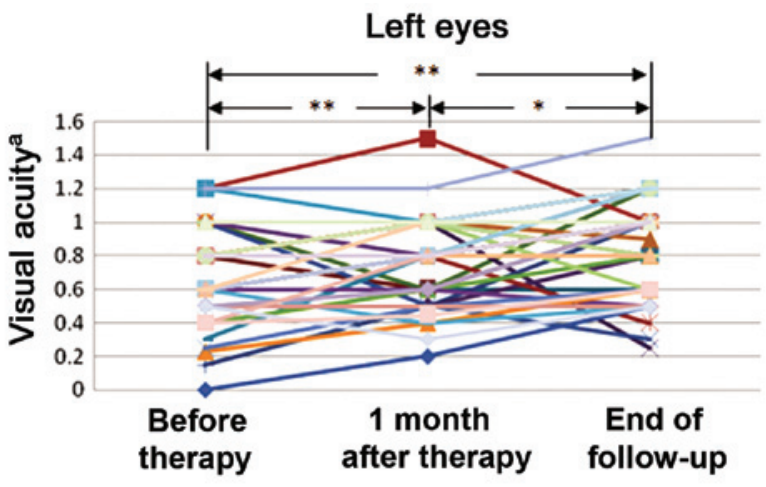

Right eyes

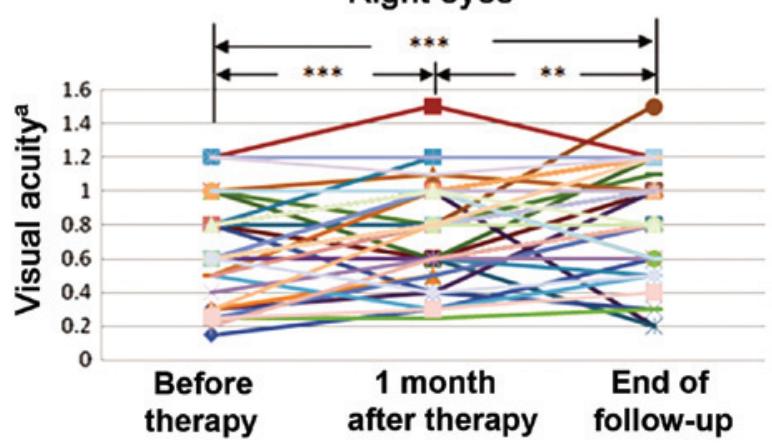

Figure 1. Alterations in visual acuity across three time points. An international standard vision chart was used to determine the visual acuity of the enrolled patients. The visual acuity of both eyes was significantly improved one month after therapy and at the end of the follow-up. ${ }^{*} \mathrm{P}<0.05,{ }^{* *} \mathrm{P}<0.01$ and ${ }^{* * *} \mathrm{P}<0.001$, as determined by repeated-measures analysis of variance.

Ocular motility was improved after treatment. Prior to treatment, 51 patients exhibited disordered ocular motility in both eyes. Grading of ocular motility was significantly reduced in their binoculus (left eye, $\mathrm{P}<0.001$; right eye, $\mathrm{P}<0.001$ ) one month after treatment, as compared with before treatment, and were also significantly reduced between one month after therapy and the end of follow-up (left eye, $\mathrm{P}<0.001$; right eye, $\mathrm{P}<0.001)$. Moreover, the enrolled patients were divided into two groups according to gender, age and smoking status, and no significant differences were detected in the interaction effect of treatment with any grouping in ocular motility.

Proptosis was reduced after treatment. Compared to baseline, the number of patients with reduced proptosis ( $\geq 2 \mathrm{~mm}$ ) was 19 $(33 \%)$ one month after therapy and $30(52 \%)$ at the end of follow up in the left eye, and 13 (22\%) one month after therapy and 27 $(47 \%)$ at the end of follow up in the right eye. Proptosis data are presented in Table III. It was demonstrated that proptosis (Fig. 2) was significantly reduced one month after therapy in the left $(\mathrm{P}<0.001)$ and right $(\mathrm{P}=0.006)$ eyes and at the end of follow-up in the left $(\mathrm{P}<0.001)$ and right $(\mathrm{P}<0.001)$ eyes. Moreover, proptosis was significantly decreased between one month after therapy and the end of follow-up in the left $(\mathrm{P}=0.005)$ and the right $(\mathrm{P}=0.001)$ eyes. No significant difference was detected in the interaction effect of treatment following grouping (gender, age and smoking status) in either eye.

CAS was reduced after treatment. CAS values were $8.24 \pm 1.81$ $(\mathrm{n}=58)$ prior to therapy, $1.98 \pm 1.79$ one month after therapy
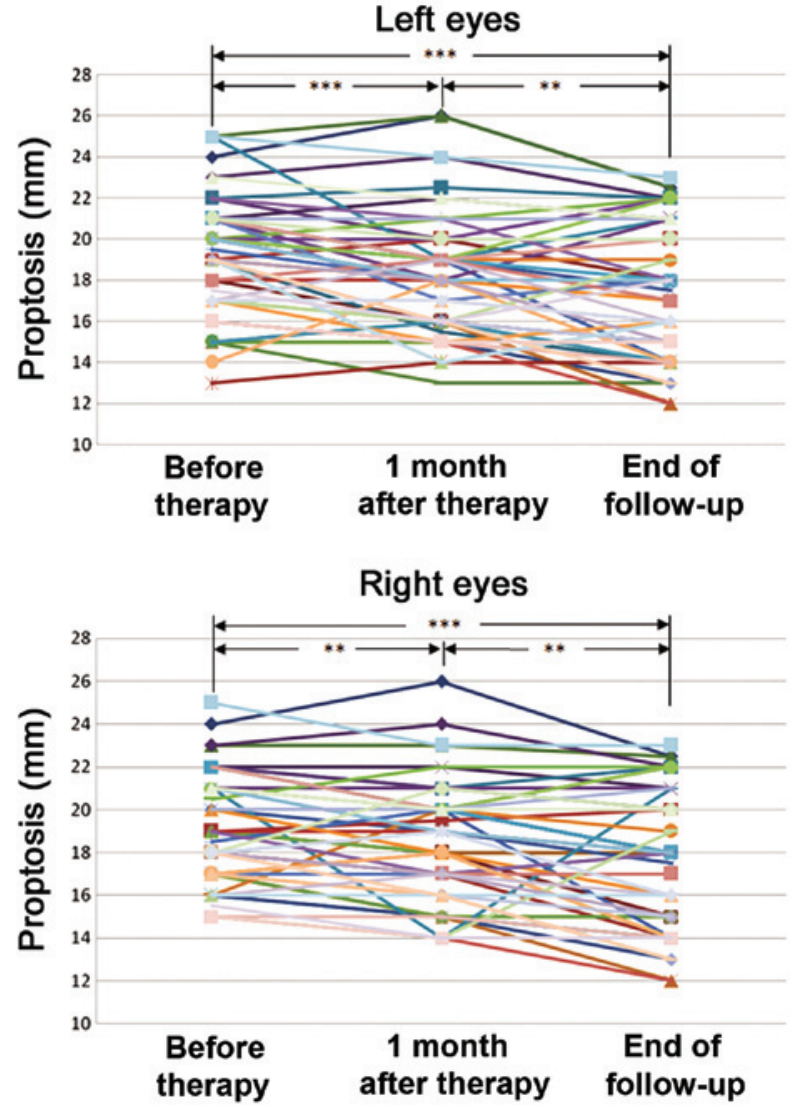

Figure 2. Alterations in the level of proptosis across three time points. The levels of proptosis in both eyes were significantly reduced one month after treatment and at the end of follow-up. ${ }^{* *} \mathrm{P}<0.01$ and ${ }^{* * *} \mathrm{P}<0.001$, as determined by repeated-measures analysis of variance.

and $1.41 \pm 1.17$ at the end of follow-up. CAS was significantly reduced one month after the therapy $(\mathrm{P}<0.001)$ and at the end of follow-up $(\mathrm{P}<0.001)$, as compared with the baseline (Fig. 3 ). No significant differences in CAS values were detected between the one month after therapy and end of follow-up time points.

Intraocular pressure was improved after treatment. Complete intraocular pressure could not be obtained for four patients. Data from the remaining 54 patients demonstrated that the intraocular pressure changed significantly with treatment in both the left $(\mathrm{P}=0.012)$ and right $(\mathrm{P}=0.046)$ eyes.

TRAb was correlated with CAS. Analysis of covariance demonstrated that the difference in serum TRAb was predominantly correlated with CAS $(\mathrm{P}=0.020)$ and was not correlated with smoking $(\mathrm{P}=0.722)$ or age $(\mathrm{P}=0.789)$. Significant differences were detected between active TAO and the baseline and after 3 cycles of treatment $(\mathrm{P}=0.033)$, but no significant difference was found after 1 or 2 cycles of treatment $(\mathrm{P}=0.722$ and $\mathrm{P}=0.155$, respectively), as compared with the baseline.

SICAM-1 levels were correlated with duration of eye disease. Analysis of covariance demonstrated that the change in sICAM-1 levels over time was correlated with the duration of eye disease $(\mathrm{P}=0.015)$ but not to CAS $(\mathrm{P}=0.912)$. Compared with the sICAM-1 level before treatment, no significant 


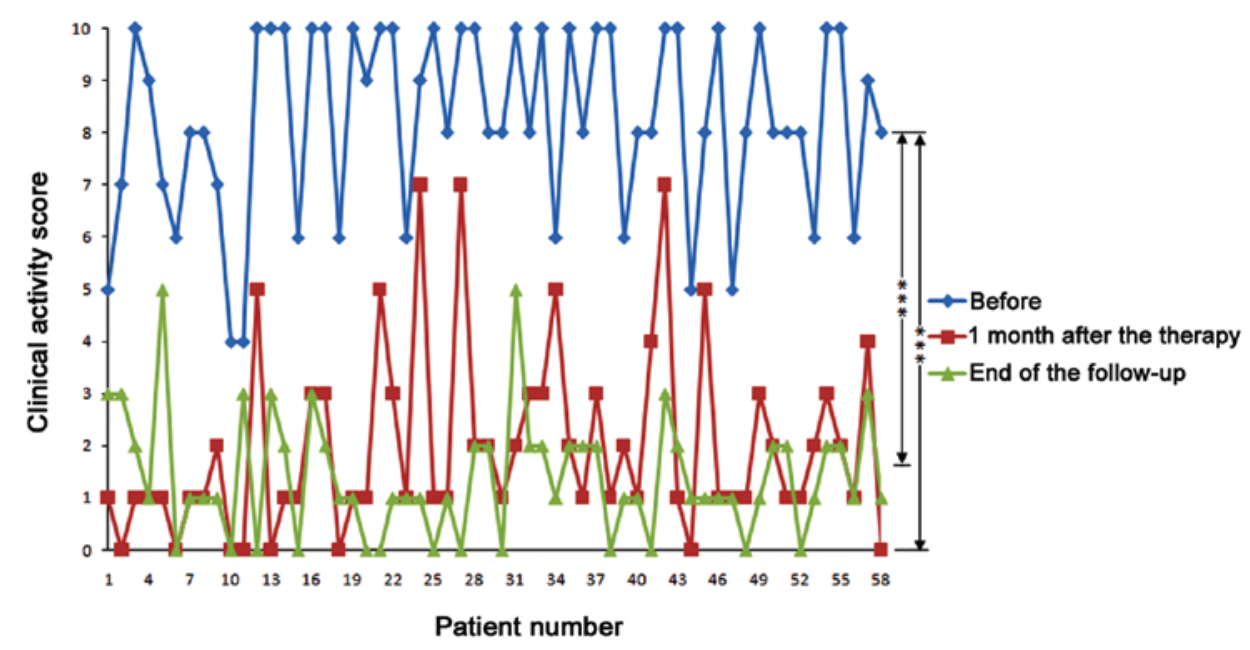

Figure 3. Alterations in clinical activity scores across three time points: before the therapy, one month after therapy and at end of the follow-up. Clinical activity scores were significantly reduced one month after treatment and at the end of follow-up. ${ }^{* * *} \mathrm{P}<0.001$, as determined by repeated measures analysis of variance.

difference was detected after 1,2 or 3 cycles of treatment $(\mathrm{P}=0.074, \mathrm{P}=0.230$ and $\mathrm{P}=0.072$, respectively).

Little adverse effects were identified after treatment. Following IVMP therapy, a female patient who was simultaneously administered fenofibrate-loaded polylactide/polyethylene glycol microspheres, suffered from severe liver injury. The patient recovered after hospitalization, during which she received liver-protecting treatment and ceased fenofibrate treatment. A male patient suffered from mildly abnormal liver function one month after IVMP therapy and fully recovered after dynamic observation for three months without any treatment. Compared with their pretreatment body weight, three females (60, 68 and 47 years old, respectively) and one male (42 years old) gained 10, 6, 5 and $10 \mathrm{~kg}$, respectively, by the end of follow-up, with no moon face or buffalo hump symptoms. Three of these patients exhibited normal body fat distribution, and one female had a large waist circumference. During the therapeutic process, blood glucose and blood pressure were monitored, and if necessary, appropriate interventions were employed for the 11 cases of hypertension and 10 cases of diabetes or impaired glucose tolerance to reduce fluctuations. No interventions for blood glucose or blood pressure were employed in the other patients, even if a rising trend was detected. Within a week the increased blood glucose and pressure were resolved in these cases. No osteoporosis, fractures, tuberculosis, serious infections, peptic ulcers or mental disorders were detected in any of the participants during the observation period.

\section{Discussion}

TAO is a chronic autoimmune disease that affects the retrobulbar tissue and has strong etiological links with autoimmune thyroid disease, which leads to blurring of vision, proptosis, extraocular muscle dysfunction, redness of the conjunctiva and pain (13). Although there are other therapies, including orbital radiotherapy and oral glucocorticoids, IVMP therapy has superior efficiency and few adverse effects in the treatment of patients with moderately to severely active TAO (13-15). In a published consensus statement $(6,16)$, the EUGOGO recommend the use of IV glucocorticoids as first-line treatment for patients with active or severe TAO.

Numerous randomized and nonrandomized trials of intravenous glucocorticosteroid have demonstrated the beneficial effects of glucocorticoids in TAO $(17,18)$, including 13 nonrandomized trials with a total of 346 patients and 10 randomized trials with a total of 234 patients. The treatment protocols for each of these studies were $0.5-1.0 \mathrm{~g}$ or $12.5-15.0 \mathrm{mg} / \mathrm{kg}$ IV methylprednisolone with or without oral prednisolone and orbital irradiation (17). Treatment with IV glucocorticoids was safe and effective in the nonrandomized (response rate, 82.6\%) and randomized (response rate, 79.9\%) trials and was associated with a lower recurrence rate and less frequent adverse effects, as compared with oral regimens (17). Ohtsuka et al (19) conducted a case-control study with 39 Japanese patients in which the first 20 patients underwent high-dose IVMP therapy followed by 24-Gy orbital radiotherapy and the remaining 19 patients only received high-dose IVMP therapy. No significant difference was detected between the two groups; suggesting that the addition of 24 Gy irradiation to IV prednisolone had no extra therapeutic benefit. In addition, the radiation may have caused radiation optic neuropathy if the cumulative dose of radiation exceeded 50 Gy or if radiation fractions of $>2$ Gy were used (20). In a survey, $91 \%$ of the responding members of the European Thyroid Association indicated that they would treat an index patient who had active and severe TAO with glucocorticoids, and $71 \%$ would immediately start with IV glucocorticoids (21). Similarly, $58 \%$ of the responding Latin-American thyroidologists would administer IV glucocorticoids (22).

The short-and long-term effects of IVMP therapy were investigated in the present report, indicating the superiority of the present study over previous studies $(17,18)$. The findings of the present study suggested that the symptoms of patients with moderately to severely active TAO may be improved or cured through IVMP therapy and that the curative effect is stable over time. It was also demonstrated that some symptoms, including 
diplopia, visual acuity, ocular motility and proptosis, may continue to improve with time after the withdrawal of therapy. No serious adverse events occurred in this carefully monitored and closely followed-up study. Serious liver injury occurred in one female patient who was taking fenofibrate-loaded polylactide/polyethylene glycol microspheres at the same time as the IVMP therapy. Her liver injury may be due to the joint impact of high-dose methylprednisolone and micronized fenofibrate. Liver injury is a serious adverse effect of high dose methylprednisolone and should be careful monitored during the course of treatment (17). Moreover, high-dose methylprednisolone should never be used with any agent that is potentially harmful to the liver. Four patients who exhibited mild weight gain over the observation period had taken oral corticosteroids for six months or more prior to treatment with IVMP. A systematic review and meta-analysis of randomized trials for TAO (23) determined that patients in the oral administration group had a high rate of steroid-related adverse events, including weight gain $(26 \%)$, hypertension (8\%) and cushingoid features $(7 \%)$, whereas patients in the IV administration group exhibited a lower rate of steroid-related events (3-4\%). Considering both the positive and adverse effects of IVMP, Zang et al (17) suggested that single doses of IV glucocorticoids should not be administered on consecutive days. An every-other-day regimen was employed in the present study.

The majority of previous studies have demonstrated that smoking is associated with an increased risk of TAO progression in Western countries and that smoking cessation may minimize the progression of TAO and improve the response to treatment (24-28). Meanwhile, a previous study in Taiwan demonstrated that no significant association with cigarette smoking was detected in male patients (29). Exploratory grouping demonstrated that smoking did not play a role in the outcomes of the present study. Smoking may not have had an effect in the present study as patients were divided according to their pretreatment status as smokers or non-smokers, and the majority of smokers stopped smoking or severely reduced their intake under the guidance of their doctors. Gender may have been a confounding factor as all of the smokers in the present study, except for one, were male, and the sample size was relatively small.

Stimulation by TRAb in the synthesis and secretion of thyroid hormone is a direct cause of Graves' disease, and TRAb is also a serum marker of Graves' disease. In the present study, the TRAb level was correlated with the activity of eye disease and may serve as a predictor of the response to methylprednisolone therapy. Treatment had no significant effect on sICAM-1 levels in the present study and sICAM-1 levels may increase during acute stress, infection and other diseases besides TAO (30-34), therefore sICAM-1 is not specific for TAO and cannot effectively reflect the changes in TAO.

In conclusion, high-dose IVMP therapy is an effective, safe and well-tolerated treatment for patients with TAO, and is associated with few adverse effects and relapses. The effects of IVMP therapy are stable, and some symptoms, including diplopia, visual acuity, ocular motility and proptosis, may continue to improve with time following treatment withdrawal. Furthermore, it was demonstrated that serum TRAb levels are correlated with the activity of TAO and may serve as a predictor of the response to methylprednisolone therapy, whereas sICAM-1 levels are not correlated. This research could provide some evidence for the treatment of moderately-to-severely active TAO and selection of IVMP schedules.

\section{Acknowledgements}

The present study was supported by the Special Project of Health Industry Research of China (grant no. 201002002).

\section{References}

1. Wall JR and Lahooti H: Pathogenesis of thyroid eye disease - does autoimmunity against the TSH receptor explain all cases? Endokrynol Pol 61: 222-227, 2010.

2. Bartley GB: The epidemiologic characteristics and clinical course of ophthalmopathy associated with autoimmune thyroid disease in Olmsted County, Minnesota. Trans Am Ophthalmol Soc 92: 477-588, 1994.

3. Brent GA: Clinical practice. Graves' disease. N Engl J Med 358: 2594-2605, 2008.

4. Kinsell LW, Partridge JW and Foreman N: The use of ACTH and cortisone in the treatment and in the differential diagnosis of malignant exophthalmos. Ann Intern Med 38: 913-917, 1953.

5. Bartalena L and Tanda ML: Clinical practice. Graves' ophthalmopathy. N Engl J Med 360: 994-1001, 2009.

6. Bartalena L, Baldeschi L, Dickinson A, Eckstein A, Kendall-Taylor P, Marcocci C, Mourits M, Perros P, Boboridis K, Boschi A, Currò N, Daumerie C, Kahaly GJ, Krassas GE, Lane CM, Lazarus JH, Marinò M, Nardi M, Neoh C, Orgiazzi J, Pearce S, Pinchera A, Pitz S, Salvi M, Sivelli P, Stahl M, von Arx G, Wiersinga WM; European Group on Graves Orbitopathy (EUGOGO). Consensus statement of the European Group on Graves' orbitopathy (EUGOGO) on management of GO. Eur J Endocrinol 158: 273-285, 2008.

7. Khoo DH, Eng PH, Ho SC, Tai ES, Morgenthaler NG, Seah LL, Fong KS, Chee SP, Choo CT and Aw SE: Graves' ophthalmopathy in the absence of elevated free thyroxine and triiodothyronine levels: Prevalence, natural history and thyrotropin receptor antibody levels. Thyroid 10: 1093-1100, 2000.

8. Khoo DH, Ho SC, Seah LL, Fong KS, Tai ES, Chee SP, Eng PH, Aw SE and Fok AC: The combination of absent thyroid peroxidase antibodies and high thyroid-stimulating immunoglobulin levels in Graves' disease identifies a group at markedly increased risk of ophthalmopathy. Thyroid 9: 1175-1180, 1999.

9. De Bellis A, Di Martino S, Fiordelisi F, Muccitelli VI, Sinisi AA, Abbate GF, Gargano D, Bellastella A and Bizzarro A: Soluble intercellular adhesion molecule-1 (sICAM-1) concentrations in Graves' disease patients followed up for development of ophthalmopathy. J Clin Endocrinol Metab 83: 1222-1225, 1998.

10. Heufelder AE and Bahn RS: Soluble intercellular adhesion molecule-1 (sICAM-1) in sera of patients with Graves' ophthalmopathy and thyroid diseases. Clin Exp Immunol 92: 296-302, 1993.

11. Dickinson AJ and Perros P: Controversies in the clinical evaluation of active thyroid-associated orbitopathy: Use of a detailed protocol with comparative photographs for objective assessment. Clin Endocrinol (Oxf) 55: 283-303, 2001.

12. Mourits MP, Koornneef L, Wiersinga WM, Prummel MF, Berghout A and van der Gaag R: Clinical criteria for the assessment of disease activity in Graves' ophthalmopathy: A novel approach. Br J Ophthalmol 73: 639-644, 1989.

13. Yang DD, Gonzalez MO and Durairaj VD. Medical management of thyroid eye disease. Saudi J Ophthalmol 25: 3-13, 2011.

14. Wakelkamp IM, Baldeschi L, Saeed P, Mourits MP, Prummel MF and Wiersinga WM. Surgical or medical decompression as a first-line treatment of optic neuropathy in Graves' ophthalmopathy? A randomized controlled trial. Clin Endocrinol (Oxf) 63: 323-328, 2005.

15. Tambe K, Bhargava J, Tripathi A, Gregory M, Burns J and Sampath R. The role of intravenous methylprednisolone immunosuppression in the management of active thyroid eye disease. Orbit 29: 227-231, 2010.

16. Bartalena L, Baldeschi L, Dickinson A, Eckstein A, Kendall-Taylor P, Marcocci C, Mourits M, Perros P, Boboridis K, Boschi A, et al: Consensus statement of the European Group on Graves' orbitopathy (EUGOGO) on management of GO. Eur J Endocrinol 158: 273-285, 2008. 
17. Zang S, Ponto KA and Kahaly GJ: Clinical review: Intravenous glucocorticoids for Graves' orbitopathy: Efficacy and morbidity. J Clin Endocrinol Metab 96: 320-332, 2011.

18. Chang TC, Kao SC, Hsiao YL, Lu CP, Huang KM and Tzeng SS Therapeutic responses to corticosteroids in Graves' ophthalmopathy. J Formos Med Assoc 95: 833-838, 1996.

19. Ohtsuka K, Sato A, Kawaguchi S, Hashimoto M and Suzuki Y: Effect of steroid pulse therapy with and without orbital radiotherapy on Graves' ophthalmopathy. Am J Ophthalmol 135: 285-290, 2003.

20. Sánchez-Orgaz M, Grabowska A, Royo-Oreja A, Asencio-Durán M, Romero-Martín R and Arbizu-Duralde A: Optic neuropathy following orbital irradiation for Graves ophthalmopathy: A case report and literature review. Orbit 31: 30-33, 2012.

21. European Group of Graves' Orbitopathy: Perros P, Baldeschi L, Boboridis K, Dickinson AJ, Hullo A, Kahaly GJ, Kendall-Taylor P, Krassas GE, Lane CM, Lazarus JH, et al: A questionnaire survey on the management of Graves' orbitopathy in Europe. Eur J Endocrinol 155: 207-211, 2006.

22. Ramos HE, Diehl LA, Camacho CP, Perros P and Graf H; Latin American Thyroid Society: Management of Graves' orbitopathy in Latin America: An international questionnaire study compared with Europe. Clin Endocrinol (Oxf) 69: 951-956, 2008

23. Stiebel-Kalish H, Robenshtok E, Hasanreisoglu M, Ezrachi D, Shimon I and Leibovici L: Treatment modalities for Graves ophthalmopathy: Systematic review and metaanalysis. J Clin Endocrinol Metab 94: 2708-2716, 2009.

24. Gillespie EF, Smith TJ and Douglas RS: Thyroid eye disease: Towards an evidence base for treatment in the 21st century. Curr Neurol Neurosci Rep 12: 318-324, 2012.

25. Hägg E and Asplund K: Is endocrine ophthalmopathy related to smoking? Br Med J (Clin Res Ed) 295: 634-635, 1987.
26. Bartalena L, Martino E, Marcocci C, Bogazzi F, Panicucci M, Velluzzi F, Loviselli A and Pinchera A: More on smoking habits and Graves' ophthalmopathy. J Endocrinol Invest 12: 733-737, 1989.

27. Bahn RS: Graves' ophthalmopathy. N Engl J Med 362: 726-738, 2010.

28. Prummel MF and Wiersinga WM: Smoking and risk of Graves' disease. JAMA 269: 479-482, 1993.

29. Chen YL, Chang TC and Chen CJ: Influence of smoking on Graves disease with or without ophthalmopathy and nontoxic nodular goiter in Taiwan. J Formos Med Assoc 93: 40-44, 1994.

30. Briassoulis G, Papassotiriou I, Mavrikiou M, Lazaropoulou C and Margeli A: Longitudinal course and clinical significance of TGF-beta1, sL- and sE-Selectins and sICAM-1 levels during severe acute stress in children. Clin Biochem 40: 299-304, 2007.

31. Zhou SL, Chen YL, Peng XZ, Zhao YX, Xie PZ, Ling L, Chen XW and Dai KM: Expression of serum sICAM-1 in patients with primary hepatocellular carcinoma and its relationship with liver fibrosis. Xi Bao Yu Fen Zi Mian Yi Xue Za Zhi 28: 181-182, 2012 (In Chinese).

32. de Pablo R, Monserrat J, Reyes E, Díaz D, Rodríguez-Zapata M, de la Hera A, Prieto A and Álvarez-Mon M: Circulating sICAM-1 and sE-Selectin as biomarker of infection and prognosis in patients with systemic inflammatory response syndrome. Eur J Intern Med 24: 132-138, 2013

33. Cha JJ, Hyun YY, Jee YH, Lee MJ, Han KH, Kang YS, Han SY and Cha DR: Plasma concentration of soluble intercellular adhesion molecule-1 (sICAM-1) is elevated in type 2 diabetic patients, and sICAM-1 synthesis is associated with leptin-induced activation of the mitogen-activated protein kinase (MAPK) pathway. Inflammation 36: 878-887, 2013.

34. Dymicka-Piekarska V, Guzinska-Ustymowicz K, Kuklinski A and Kemona H: Prognostic significance of adhesion molecules (sICAM-1, sVCAM-1) and VEGF in colorectal cancer patients. Thromb Res 129: e47-e50, 2012. 